(RESEARCH ARTICLE)

\title{
Pattern of anti-malarial drugs and artemether combination therapy adherence in an institution based medical centre, Nigeria
}

\author{
Ujuamala Uloma Ezeani ${ }^{1}$, Penaere Theresa Osahon ${ }^{2}$ and Michael Chukwudi Ezeani ${ }^{3, *}$ \\ ${ }^{1}$ Department of pharmacology and toxicology, faculty of pharmaceutical sciences, Nnamdi Azikiwe University Awka, \\ Anambra state, Nigeria. \\ ${ }^{2}$ Department of Clinical Pharmacy and Pharmacy Practice, Faculty of Pharmacy, University of Benin, Nigeria, \\ ikponmwosa. \\ ${ }^{3}$ Immunology Department, Faculty of Medicine, Nnamdi Azikiwe University, Awka, Anambra state, Nigeria.
}

Publication history: Received on 12 November 2020; revised on 22 November 2020; accepted on 25 November 2020

Article DOI: https://doi.org/10.30574/wjarr.2020.8.3.0437

\begin{abstract}
The change in policy guidelines for treating uncomplicated malaria became necessary because the therapeutic efficacy of chloroquine and SP had deteriorated. Hence compliance is a necessity to enable effective check on malaria. This work was carried out to evaluate antimalaria drug prescription and to update its usage in line with WHO guideline on Artemeter Combination therapy in a university based medical center. We utilized descriptive, cross-sectional, retrospective study of antimalaria prescriptions purposely carried out among male and female outpatients with mean age of $22.4 \pm 2.8$ at a University health facility. This comprised all outpatients prescriptions that contained at least one antimalarial drug filed from October 2018 to September 2019. Systematic sampling was used to select the prescriptions. Based on the total number of 1250 prescriptions containing at least one antimalarial drug, a sampling interval of 5 was calculated and simple balloting was used for the first pick. A total number of two hundred and fifty (250) prescriptions containing at least one antimalarial drug were selected for the study. Out of 250 antimalaria prescriptions, usage of ACT class of Artemeter lumefantrine, Artemeter Amodiaquine and Artemeter Piparaqiune were recorded at 45.6\%, 10.4\% and $9.6 \%$ respectively. Triple combination Artemeter lumefantrine and Sulphadoxine-Pyrimethamine was recorded at $20.4 \%$ while Sulphadoxine-Pyrimethamine was recorded at $4 \%$. Combination of antimalarial drugs with antibiotics was recorded at $31.2 \%$. This study showed compliance with National Antimalarial Treatment Guideline for the treatment of malaria infection as it regards the use of artemisinin-based combination therapy. The frequency usage of artemeter lumefantrine was proceeding among other ACTs. The frequency in co-prescription of antibiotics with anti-malaria should be guarded to comply with WHO recommendation.
\end{abstract}

Keywords: Anti- malarial; Prescription Pattern; Policy Adherence; Clinical Responses Artemether Combination

\section{Introduction}

Globally, Half the world's population, about 3.4 billion people in 92 countries are at risk of being infected with malaria and developing disease, and 1.1 billion are at high risk (>1 in 1000 chance of getting malaria in a year). According to the World Malaria Report 2018, there were 219 million cases of malaria globally in 2017 (uncertainty range 203-262 million) and 435000 malaria deaths, representing a decrease in malaria cases and deaths rates of $18 \%$ and $28 \%$ since 2010, respectively. The burden was heaviest in the World Health organization (WHO) African Region, where an estimated $93 \%$ of all malaria deaths occurred and indicating increased burden of malaria in African region since 2010. Children aged less than 5 years, accounted for $61 \%$ of all deaths [1]. Increasing resistance of the parasite to currently available drugs has created an urgent need to discover new treatments [2]. Mosquitoes are found around the globe,

\footnotetext{
${ }^{*}$ Corresponding author: Michael Chukwudi Ezeani

Immunology Department, Faculty of Medicine, Nnamdi Azikiwe University, Awka, Anambra state, Nigeria. 
while incidents of malaria are concentrated in the tropics. Even if more Anopheles are found in the tropics, due to their faster development in temperate water, this still does not fully explain historical accounts in which malaria is reported in some regions earlier in more ancient time than others. Thirty countries in Sub-Saharan Africa account for $90 \%$ of global malaria deaths. Nigeria, Democratic Republic of Congo (DRC), Ethiopia, and Uganda account for nearly $50 \%$ of the global malaria deaths. Malaria is the 2nd leading cause of death from infectious diseases in Africa, after HIV/AIDS. Almost 1 out of 5 deaths of children under 5 in Africa are due to malaria. Malaria is a major public health problem in Nigeria where it accounts for more cases and deaths than any other country in the world. Malaria is a risk for $97 \%$ of Nigeria's population [1]. The remaining 3\% of the population live in the malaria free highlands. There are an estimated 100 million malaria cases with over 300,000 deaths per year in Nigeria. This compares with 215,000 deaths per year in Nigeria from HIV/AIDS. Malaria contributes to an estimated 11\% of maternal mortality (WHO, 2018) [1].

Malarial infection begins when a person is bitten by an infected female anopheles mosquito and Plasmodium species parasites in the form of sporozoites are injected into the bloodstream. The sporozoites travel to the liver, multiplying asexually over the next 7-10 days (Mawson, 2013) [3]. During this time there are no symptoms. The parasites, now in the form of merozoites, emerge from the liver cells in vesicles and travel through the heart to the capillaries of the lungs. The vesicles eventually disintegrate, releasing the merozoites to enter the bloodstream where they invade and multiply in erythrocytes. When the cells burst, the parasites invade more erythrocytes [3]. Clinical symptoms, including fever, occur in synchrony with the rupture of infected erythrocytes and the release of erythrocyte and parasite debris, including malarial pigment (hemozoin) and glycophosphatidylinositol, the putative 'malaria toxin. As noted, the symptoms are associated with the rupture of the infected erythrocytes and the release of putative malaria toxins, which activate peripheral blood mononuclear cells and stimulate the release of cytokines. It is believed that the balance between pro-inflammatory and anti-inflammatory cytokines, chemokines, growth factors, and effector molecules determines disease severity [4] Studies have reported increased IL-1B, IL-6, IL-8, and TNF-alpha in late-onset severe disease, and a low IL-10: TNF-alpha ratio. The role of cytokines, however, remains contradictory and unclear [5]. Alterations in retinoids (vitamin A and its congeners) also occur in malaria, but the precise role of retinoids in the disease may be different and even opposite to the traditional focus on vitamin A deficiency and supplementation. From previous researches done on the pathogenesis of malaria, there is a suggestion that an endogenous form of hypervitaminosis A induced by the parasite contributes significantly to the signs and symptoms of the disease [5]. Plasmodium falciparum selectively absorbs vitamin A from the host and appears to use it for its metabolism; serum vitamin A levels are also reduced in children with malaria. Although vitamin A is essential in low concentration for numerous biological functions, higher concentrations are cytotoxic and pro-oxidant, and potentially toxic quantities of the vitamin are stored in the liver. During their life cycle in the host the parasites remain in the liver for several days before invading the red blood cells (RBCs). The hypothesis proposed is that the parasites emerge from the liver packed with vitamin $A$ and use retinoic acid (RA), the main biologically active metabolite of vitamin A, as a cell membrane destabilizer to invade the RBCs throughout the body [5].

United States Malaria Initiative, supports four scientifically proven key interventions to prevent and treat malaria: 1) the promotion of insecticide treated mosquito nets (ITNs); 2) indoor residual spraying (IRS); 3) intermittent preventive treatment for pregnant women (IPT); and 4) diagnosis and treatment. Prevention programs focus on the distribution and use of bed nets, called Long Lasting Insecticidal Nets (LLINS), including evidence-based health communication programs on the mode of malaria transmission and the importance of sleeping under ITNs. [6]. Indoor Residual Spraying (IRS) involves the coordinated, timely spraying of the interior walls of homes with insecticides that kill mosquitoes. Intermittent preventive treatment for pregnant women (IPTp) is an effective means of reducing the effects of malaria in both the pregnant woman and her unborn child by giving at least two doses of the drug sulfadoxine-pyrimethamine (SP). Prompt parasitological confirmation by microscopy or Rapid Diagnostic Test (RDT) is recommended for all patients with suspected malaria before treatment begins. Artemisinin-based combination therapy (ACT) has become the standard treatment of uncomplicated malaria [6]. In addition to the above, malaria can also be prevented by taking several simple precautions such as preventing mosquito bites by using covered clothes and mosquito repellents. Awareness of the risk of malaria in high risk zones is the first step to preventing malaria. Thereafter prescription of antimalarial tablet to prevent contracting malaria should be encouraged while travelling to these malaria endemic zone. Immediate diagnosis and treatment can help prevent complications and death.

Malaria can be prevented or treated with medications. The type and duration of drugs depends on the type of malaria, its severity and the condition of the patient (e.g pregnancy). Based on WHO recommendation, children and adult with uncomplicated Plasmodium .falciparium (except pregnant women in their first trimester) should be treated with one of the following artemisinin-based combination therapies (ACT): Artemether + lumefantrine; Artesunate + amodiaquine; Artesunate + mefloquine; Dihydroatemisinine + piperaquine; Artesunate + sulphadoxine-pyrimethamine. These ACTs regimen should provide three days treatment with artemisinin derivatives [7]. Recommendations of WHO for the treatment of complicated (severe) malaria (including infants, pregnant women in all trimesters and lactating mothers), 
encourages the use of intravenous or intramuscular artesunate for at least 24 hours and until they can tolerate oral medication. When oral ACT is started, the treatment should be completed for three days (single dose of primaquine should be added in areas of low transmission). If artesunate is not available, the use of injection artemether should be encouraged in preference to quinine in treating adult and children with severe malaria. Two landmark trials in patients with severe malaria definitively showed that intravenous artesunate reduced mortality by 35 and $23 \%$ in adults and children, respectively, compared to quinine [8,9]. Intravenous artesunate is now the first-line treatment for severe malaria as recommended by the WHO. Artemether and quinine are the second-line therapies [7]. The mechanism of improved survival over quinine is the rapid cidal activity of artesunate on young ring forms, preventing parasite maturation and sequestration [10].

The Nigerian government has recently changed its policy guidelines for treating uncomplicated malaria to artemetherlumefantrine or amodiaquine-artesunate replacing monotherapy of chloroquine and sulfadoxine-pyrimethamine (SP). The policy change became necessary because the therapeutic efficacy of chloroquine and SP had deteriorated [11]. Any introduction of new treatments will require evidence from audit to understand current prescribing practices, and training to provide guidance [12]. The success of a new treatment policy would depend on the adherence of health providers and patients to the recommendations [13]. Anti-malarial drug resistance is a major public health problem which hinders the control of malaria. Malaria being a major health problem in the country as well as the increasing emergence of resistance to the conventional anti-malarial therapy, it is necessary to check for the prescribing pattern of the anti-malarial drugs in inpatient settings in the health care system.

Prescription practices have been shown to influence the emergence of resistance to antimalarial drugs, thus the success of a new treatment policy would depend on adherence of health providers and patients to treatment recommendation $[14,15]$. This is important in other to protect the clinical shelf life of artemisinin based combinations, since they remain the most valuable drugs currently available in the treatment of malaria (Gbotosho et al., 2009) [16]. Rational use of drugs against most common and life-threatening tropical diseases such as malaria remains a huge challenge, particularly in sub-Saharan African region. (Breman etal, 2004) [17]. This region carries a disproportionately high share of the global malaria burden. They contribute an estimated $90 \%$ of global episodes of clinical malaria and $90 \%$ of global malaria mortality. (WHO, 2010a; WHO, 2010b) $[18,19]$. Appropriate treatment of malaria and the correct use of antimalarial drugs are needed in order to achieve Nigerian's goal of pre-elimination and reducing malaria related death to zero. Drug use studies could unravel drug use problems and give feedback to the prescriber to create awareness on rational use of drugs [20]. The success of any treatment policy depends largely on the adherence of health care providers to treatment recommendations based on policy statements. Hence antimalarial prescriptions by prescribers are expected to be influenced by the change in the National antimalarial policy in other to improve the trend in malaria morbidity and mortality. Therefore the objective of this study is of paramount importance to evaluate the prescriptions of antimalarial medicines and to ascertain the adherence of prescribers to the National Antimalarial Treatment Guideline in the treatment of malaria infections, the most antimalarial prescribed in this facility and the efficacy of the antimalarial used.

\section{Material and methods}

\subsection{Study site and design}

This was a descriptive, cross-sectional, retrospective study of prescriptions purposely carried out among outpatients at a University health facility in Southeast Nigeria. These outpatients were seen by medical doctors and they access their approved drugs with a total payment from the pharmacy unit. The facility is a university medical center that renders health services to students, staffs and the public.

\subsection{Data Collection}

This comprised all outpatients prescriptions that contained at least one antimalarial drug filed from October 2018 to September 2019. Large sample size which exceeded the minimum of 100 suggested by WHO was employed in order to enhance the reliability of the results since only one health facility was used for the study (Okoro and Jamiu, 2018)[22]. The patients were asked to come back after 3 days for clinical response assessment. Systematic sampling was used to select the prescriptions. Based on the total number of 1250 prescriptions containing at least one antimalarial drug, a sampling interval of 5 was calculated and simple balloting was used for the first pick. At the end, a total number of two hundred and fifty (250) prescriptions containing at least one antimalarial drug were selected for the study [21]. All prescriptions that have at least one antimalarial drug in it were selected, while all antimalarial prescriptions for pregnant women were excluded. 


\subsection{Data Analysis}

Data were later keyed into Statistical Package for Social Sciences (SPSS, version 23, Chicago, USA) and coded for analysis. Raw data were double-checked with soft data for consistency. Descriptive statistics (frequency, percentage and mean \pm SD) were performed for distribution patterns. Chi-square was used to determine the significant association of Norminal variables. Statistical significance was set as a value less than $0.05(\mathrm{P}<0.05)$ at $95 \%$ confidence interval.

\section{Results}

\subsection{Frequency and Pattern of Prescription of Antimalaria Drugs}

Out of the two hundred and fifty prescriptions, 51(20.4\%) were Artemeter Lumefatrine / Sulphadoxine-Pyrimethamine, 114(45.6\%) were Artemeter Lumefatrine, 26(10.4\%) were Artemeter Amodiaquine, 24(9.6\%) were Artemeter Piparaquine, 12(4.8\%) were Artemeter, 12(4.8\%) were Artemeter /Sulphadoxine-Pyrimethamine, 4(1.6\%) were Sulphadoxine-Pyrimethamine and 7(2.8\%) were without antimalaria drug prescription (Table 1).

Table 1 Frequency and Pattern of Prescription of Antimalaria Drugs.

\begin{tabular}{|l|c|l|}
\hline Antimalarial Drugs & Prescription Frequency & Percentage \\
\hline Artemeter Lumefatrine / Sulphadoxine-Pyrimethamine & 51 & 20.4 \\
\hline Artemeter Lumefatrine & 114 & 45.6 \\
\hline Artemeter Amodiaquine & 26 & 10.4 \\
\hline Artemeter Piparaquine & 24 & 9.6 \\
\hline Artemeter & 12 & 4.8 \\
\hline Artemeter /Sulphadoxine-Pyrimethamine & 12 & 4.8 \\
\hline Sulphadoxine-Pyrimethamine & 4 & 1.6 \\
\hline No antimalaria & 7 & 2.8 \\
\hline
\end{tabular}

\subsection{Frequency Distribution of Various Co-prescribed Drugs with Antimalaria}

Out of the 250 prescriptions, some of the essential drugs co-prescribed with antimalaria drugs include antibiotics, vitamin c, paracetamol, Multivitamins, diclofenac and piriton. The total numbers of antibiotics (78 times), vitamin c (19 times), diclofenac (42 times) and piriton (86 times) were significantly low, with $\mathrm{P}=0.002,0.004,0.011$ and 0.014 respectively while total numbers of paracetamol and multivitamin were significantly high with $\mathrm{P}=0.000$ and 0.000 respectively.

The distribution of these essential drugs co-prescribed with antimalaria drugs showed that antibiotics were coprescribed 26 times with Artemeter Lumefantrine - Sulphadoxine-Pyrimethamine, 4 times with Artemeter Lumefantrine, 27 times with Artemeter Amodiaquine, 3 times with Artemeter Piparaquine, 2 times with Artemeter, 8 times with Artemeter-Sulphadoxine-Pyrimethamine, 2 times with Sulphadoxine-Pyrimethamine and 6 time amongst those without any antimalaria drug prescription.

The distribution of these essential drugs co-prescribed with antimalaria drugs showed that vitamin c was co-prescribed 11 times with Artemeter Amodiaquine, 2 times with Artemeter and 6 times with Artemeter-SulphadoxinePyrimethamine.

The distribution of these essential drugs co-prescribed with antimalaria drugs showed that paracetamol was coprescribed 39 times with Artemeter Lumefantrine - Sulphadoxine-Pyrimethamine, 5 times with A Artemeter Lumefantrine, 108 times with Artemeter Amodiaquine, 23 times with Artemeter Piparaquine, 7 times with Artemeter, 24 times with Artemeter-Sulphadoxine-Pyrimethamine, 4 times with Sulphadoxine-Pyrimethamine and 10 times amongst those without any antimalaria drug prescription. 
The distribution of these essential drugs co-prescribed with antimalaria drugs showed that multivitamins were coprescribed 43 times with Artemeter Lumefantrine - Sulphadoxine-Pyrimethamine, 7 times with Artemeter Lumefantrine, 99 times with Artemeter Amodiaquine, 20 times with Artemeter Piparaquine, 4 times with ArtemeterSulphadoxine-Pyrimethamine, 21 times with Artemeter, 4 times with Sulphadoxine-Pyrimethamine and 42 times amongst those without any antimalaria drug prescription.

The distribution of these essential drugs co-prescribed with antimalaria drugs showed that diclofenac was coprescribed 5 times with Artemeter Lumefantrine - Sulphadoxine-Pyrimethamine, 2 times with Artemeter Lumefantrine, 20 times with Artemeter Amodiaquine, none with Artemeter Piparaquine, 2 times with Artemeter, 9 times with Artemeter-Sulphadoxine-Pyrimethamine, none with Sulphadoxine-Pyrimethamine and 4 times amongst those without any antimalaria drug prescription.

The distribution of these essential drugs co-prescribed with antimalaria drugs showed that piriton was co-prescribed 22 times with Artemeter Lumefantrine - Sulphadoxine-Pyrimethamine, 5 times with Artemeter Lumefantrine, 45 times with Artemeter Amodiaquine, 6 times with Artemeter Piparaquine, 2 times with Artemeter, 4 times with ArtemeterSulphadoxine-Pyrimethamine, none with Sulphadoxine-Pyrimethamine and 2 time amongst those without any antimalaria drug prescription (Table 2).

Table 2 Frequency Distribution of Various Co-prescribed Drugs with Antimalaria.

\begin{tabular}{|c|c|c|c|c|c|c|}
\hline Antimalaria & Antibiotic & Vitamin C & Paracetamol & Multivit & Diclofenac & Piriton \\
\hline $\mathrm{AL} / \mathrm{SP}$ & 26 & 0 & 39 & 43 & 5 & 22 \\
\hline A L & 4 & 0 & 5 & 7 & 2 & 5 \\
\hline $\mathrm{AA}$ & 27 & 11 & 108 & 99 & 20 & 45 \\
\hline A P & 3 & 0 & 23 & 20 & 0 & 6 \\
\hline Artemeter & 2 & 2 & 7 & 4 & 2 & 2 \\
\hline Artemeter /SP & 8 & 6 & 24 & 21 & 9 & 4 \\
\hline SP & 2 & 0 & 4 & 4 & 0 & 0 \\
\hline No antimalaria & 6 & 0 & 10 & 12 & 4 & 2 \\
\hline Total & 78 & 19 & 220 & 210 & 42 & 86 \\
\hline P.value & 0.002 & 0.004 & 0.000 & 0,000 & 0.011 & 0.014 \\
\hline
\end{tabular}

KEYS: AL/SP= Artemether + Lumefantrine and Sulphadoxine/pyrimethamine; $\mathrm{AL}=\mathrm{Artemether}+$ Lumefantrine; $\mathrm{AA}=\mathrm{Artesunate}+\mathrm{Amodiaquine}$; $\mathrm{AP}=$ Artesunate + piperaquine; Artemeter $/ \mathrm{SP}=$ Artemether and Sulphadoxine/pyrimethamine; $\mathrm{SP}=\mathrm{Sulphadoxine/pyrimethamine}$

\subsection{Post Prescription Clinical Response from the Subjects after 3 Days of Drug Usage}

Post prescription clinical response from the subjects treated with antimalaria drugs after 3 days of drugs usage showed that:

Out of 114 subjects under Artemeter Lumefantrine, 94 presented without symptom, 15 did not return for feedback, 5 presented with symptom after the 3 days. The number of subjects under Artemeter Lumefantrine, that presented without symptoms after 3 days of drug usage was significantly high $\mathrm{P}=0.000$

Out of the 26 subjects under Artemeter Amodiaquine, 17 presented without symptom, 5 did not return to give feedback, 4 presented with symptom after the 3 days. The number of subjects under Artemeter Amodiaquine, that presented without symptoms after 3 days of drug usage was not significantly high $\mathrm{P}=0.914$.

Out of the 23 subjects under Artemeter Piparaquine, 14 presented without symptom, 5 did not return to give feedback, 4 presented with symptom after the 3 days. The number of subjects under Artemeter Piparaquine, that presented without symptoms after 3 days of drug usage was not significantly high $\mathrm{P}=0.751$.

Out of the 51 subjects under Artemeter Lumefantrine- Sulphadoxine-Pyrimethamine, 26 presented without symptom, 16 did not return to give feedback, 9 presented with symptom after the 3 days. The number of subjects under Artemeter 
Lumefantrine- Sulphadoxine-Pyrimethamine, that presented without symptoms after 3 days of drug usage was significantly high $\mathrm{P}=0.021$.

Out of the 4 subjects under Sulphadoxine-Pyrimethamine, 4 presented with symptom after the 3 days. The number of subjects under Sulphadoxine-Pyrimethamine,that presented with symptoms after 3 days of drug usage was significantly high $\mathrm{P}=0.000$ (Table 3.).

Table 3 Post Prescription Clinical Response from the Subjects after 3 Days of Drug Usage.

\begin{tabular}{|l|c|c|c|c|}
\hline & No symptoms after 3 days & No feedback after 3 days & Symptoms after 3 days & P-value \\
\hline AL (N=114) & 94 & 15 & 5 & 0.000 \\
\hline AA (N=26) & 17 & 5 & 4 & 0.914 \\
\hline AP (N=24) & 15 & 5 & 4 & 0.751 \\
\hline AL/SP(N=51) & 26 & 0 & 9 & 0.021 \\
\hline SP(N=4) & 0 & 3 & 4 & 0.000 \\
\hline Artemether (N=12) & 5 & 2 & 1 & 0.142 \\
\hline Artemether/SP(N=12) & 9 & 0 & 0.933 \\
\hline
\end{tabular}

KEYS: $\mathrm{AL}=$ Artemether + Lumefantrine; $\mathrm{AA}=$ Artesunate + Amodiaquine; $\mathrm{AP}=$ Artesunate + piperaquine; $\mathrm{AL} / \mathrm{SP}=\mathrm{Artemether}+\mathrm{Lumefantrine}$ and Sulphadoxine/pyrimethamine; $\mathrm{SP}==$ Sulphadoxine/pyrimethamine; Artemether/SP== Artemether and Sulphadoxine/pyrimethamine

\subsection{Demographic characteristics of Patients Under antimalarial drugs prescription}

The demographic records of the subjects under the prescription showed that 89 (35.6\%) were male while 161 (64.4\%) were female. The mean age of the male subjects was 23.2 2 2.6. Minimum age recorded for male was 18 while the maximum age for male was 29. On the other hand, the mean age of the female subjects was 22.4 \pm 2.8 . Minimum age recorded for female was 18 while the maximum age for female was 32 (Table 4).

Table 4 Demographic characteristics of Patients Under antimalarial drugs prescription

\begin{tabular}{|l|l|l|l|}
\hline Sex & Frequency & Percentage & \\
\hline Male & 89 & 35.6 & \\
\hline Female & 161 & 64.4 & \\
\hline Total & 250 & 100 & \\
\hline & & & \\
\hline Age & Mean & Minimum & Maximum \\
\hline Male (N=89) & $23.2 \pm 2.6$ & 18 & 29 \\
\hline Female & $21.9 \pm 2.8$ & 18 & 32 \\
\hline Total & $22.4 \pm 2.8$ & & \\
\hline
\end{tabular}

\section{Discussion}

This work revealed prevalent choice of AL among other ACTs. Other frequently used ACTs revealed are atesunateamogiaquine and atesunate-piparaquine respectively. This finding is in line with the results of the drug efficacy trials carried out in all the geopolitical regions of the country in 2004 which found AL to be highly efficacious and thus suitable for use in the treatment of uncomplicated P. falciparum malaria in the country. However an increase in the coprescription of Artemether-Lumefantrine with- Sulphadoxine-Pyrimethamine (SP) was observed. Sulfadoxinepyrimethamine is an anti-malaria drug currently recommendation by WHO for all women in malaria-endemic areas in Africa for intermittent preventive treatment (IPT), as part of their antenatal care (Tarning, 2016) [22]. Unfortunately, the effectiveness of sulfadoxine-pyrimethamine is challenged by widespread drug resistance in many areas [22]. The 
reason for this combination could not be ascertained but information obtained showed that such combination is made when resistance to ACTs is suspected. The new artemisinin-based combination therapy (ACT) was introduced in 2005 with Artemether-Lumefantrine (AL) as first-line treatment for uncomplicated malaria and Artesunate + Amodiaquine (copackaged) as alternative [23]. In line with this, this work was conducted to review the prescription pattern of antimalaria drugs in a University health facility in Southeast Nigeria, considering the importance of adherence to the policy guidelines by healthcare providers and patients as developed by World Health Organization in 2006 and in 2010, with the intention to encourage and update compliance to the policy. Prescription of sulfadoxine-pyrimethamine alone was found at significant minimal, moreover $100 \%$ resistance was recorded in subjects with such prescription. Suffice it to say that malaria parasite resistance to sulfadoxine-pyrimethamine is still upheld.

Considering the Essential Drug List (EDL), antibiotics, vitamin C, paracetamol, multivitamins, diclofenac and piriton were frequently recorded. Combination of antimalarial drugs with antibiotics was found to be significantly low, but revealed a higher value of $31.2 \%$ as against the WHO reference range of $20.0 \%-26.8 \%$ [24]. Other NHIS studies have also reported values above WHO reference range $[25,26]$. Essentially, prescription of antimalarial drugs with antibiotics could occur to take care of any potential coinfection or to prevent undetectable infection showing up [27]. However, it may imply that the prescriber is not too certain about the real diagnosis. Overuse of antibiotics may lead to emergence of drug resistant microorganisms and increased side effects and cost of healthcare. There has been no established need for antimalaria co-prescription with antibiotics apart from the reasons mentioned above. Therefore it could be efforts in futility and increasing the cost of treatment. Should such combination occur, it is important to adhere to WHO recommendation.Percentage prescription of Vitamin C in combination with ACT was found to be low at $7.6 \%$ which indicates adherence to restriction of co-prescription of vitamin c with ACTs. However, one would think that such combination should not occur at all. It is important to review the usefulness of Vitamin C in malaria treatment. Ascorbic acid has antioxidant properties and is reported to mop up free radicals (Marwaha, 2016) [28]. Since malaria infection imposes tremendous oxidative stress on the host, the antimalarials are often prescribed with vitamin C or similar antioxidant supplements. However, the antioxidant effect in erythrocytes has been reported to depend upon the presence or absence of glutathione. In the presence of glutathione, ascorbic acid has synergistic antioxidant activity against haem-mediated cell toxicity [28, 29]. In glutathione deficient red cells, as often happens in parasitized RBCs due to oxidative stress, ascorbic acid can react with iron or iron containing compounds to generate hydrogen peroxide or hydroxyl radical and accentuate the haemolytic mechanisms in malaria [28, 29]. Vitamin C may have additional detrimental effects in malaria. Results from an experimental study have shown that concurrent administration of artemether and ascorbic acid compromised the rates of parasite clearance in P. berghei malaria infection in mice. This effect was more pronounced at higher doses of ascorbic acid. The high doses of vitamin $\mathrm{C}$ by itself could inhibit growth of malarial parasite to some extent (Marwaha, 2016) [28]. Based on this, it is still currently not recommended to prescribe ACTs with vitamin C. Increased co-prescription of the drugs under the essential drug list was prevalent with Artemether-Amodiaquine. And the reason could not be established. To link it with the reported hypoglycemic side effect of Artemether-Amodiaquine requires further work and analysis. The use of Artemether-Lumefantrine was found in this study to be of high potency and efficacy. This coiled from the significant increase in the number of subjects with positive clinical response after 3 days of the drug usage.

\section{Conclusion}

The frequent usage of artemeter lumefantrine was preceding among other ACTs. This study showed compliance with National Antimalarial Treatment Guideline for the treatment of malaria infection as it regards the use of artemisininbased combination therapy. The frequency in co-prescription of antibiotics with anti-malaria was recorded to be higher than the WHO recommendation.

\section{Recommendation}

The use of ACT in combination with SP should be clinically evaluated. It is recommended that laboratory tests should be carried out to confirm or rule out bacterial infections before antibiotic co-prescription with artemisinin-based combination therapy. The prescription guideline should farther be adhered to.

\section{Compliance with ethical standards}

\section{Acknowledgments}

We acknowledge the permission given to us by the management of Nnamdi Azikiwe Medical Center Nnewi Campus and cooperation of the pharmaceutical unit of the Center during data collection. 


\section{Disclosure of conflict of interest}

We declare that there is no conflict of interest trailing this work.

\section{Statement of informed consent}

Post case computational data of patients attending clinic at the medical center were used.

\section{References}

[1] World Health Organization World Malaria Report at glance. 2018.

[2] Crawley J, Chu C, Mtove G, Nosten F. Malaria in children. Lancet. 2010; 375: 1468-1481.

[3] Mawson AR.The pathogenesis of malaria: a new perspective.Pathogens global health. 2013; 107(3): 122-129.

[4] Langhorne J, Ndungu FM, Sponaas AM, Marsh K. Immunity to malaria: more questions than answers. Nature Immunology. 2008; 9(7): 725-732.

[5] Perkins DJ, Were T, Davenport GC, Kempaiah P, Hittner JB, Ong'echa JM. Severe malarial anemia: innate immunity and pathogenesis. International Journal of Biological Sciences. 2011; 7: 1427-1442.

[6] United States Embassy in Nigeria Economic Section [Internet]. 2011. Available from https://ng.usembassy.gov/business/economic-data-reports-nigeria/

[7] World Health Organization Guidelines for the treatment of malaria. 3rd edition. Geneva: World Health Organization; 2015. p. 31-71.

[8] Dondorp AM, Nosten F, Stepniewska K, Day N, White N. Artesunate versus quinine for treatment of severe falciparum malaria: a randomized trial. Lancet. 2005; 366: 717-725.

[9] Dondorp AM, Fanello CI, Hendriksen IC, Gomes E, Seni A, Chhaganlal KD, Bojang K, Olaosebikan R, Anunobi N, Maitland K, Kivaya E, Agbenyega T, Nguah SB, Evans J, Gesase S, Kahabuka C, Mtove G, Nadjm B, Deen J, MwangaAmumpaire J, Nansumba M, Karema C, Umulisa N, Uwimana A, Mokuolu OA, Adedoyin OT, Johnson WB, Tshefu AK, Onyamboko MA, Sakulthaew T, Ngum WP, Silamut K, Stepniewska K, Woodrow CJ, Bethell D, Wills B, Oneko M, Peto TE, von Seidlein L, Day NP, White NJ. Artesunate versus quinine in the treatment of severe falciparum malaria in African children (AQUAMAT): an open-label, randomised trial. Lancet. 2010; 376: 1647-1657.

[10] Ter Kuile F, White NJ, Holloway P, Pasvol G, Krishna S. Plasmodium falciparum: in vitro studies of the pharmacodynamic properties of drugs used for the treatment of severe malaria. Experimental Parasitology. 1993; 76(1): 85-95.

[11] Federal Ministry of Health. National antimalarial treatment policy [Internet]. Abuja, Nigeria. 2005. Available from http://africanchildforum.org/clr/policy\%20per\%20country/nigeria/nigeria_malaria_2005

[12] Shretta R, Omumbo J, Rapuoda B, Snow RW. Using evidence to change antimalarial drug policy in Kenya. Tropical Medicine of International Health. 2000; 6: 755-764.

[13] Zurovac D, Rowe AK, Ochola SA, Noor AM, Midia B, English M, Snow RW. Predictors of the quality of health worker treatment practices for uncomplicated malaria at government health facilities in Kenya. International Journal of Epidemiology. 2004; 33: 1080-1091.

[14] Yousif MA, Adeel AA Antimalaria prescription pattern in Gezira States :precepts and practices. East Mediterranean Health. Journal. 2000; 6: 939-947.

[15] Builders MI , Degge H, Peter JY, Ogbole E Prescription pattern of antimalarial drugs in a teaching hospitals in Nigeria. British biomedical bulletin. 2014; ISSN 2347-5447.

[16] Gbotosho GO, Happil CT, Ganiyu A, Ogundahunsi OA, Sowunmi A, Oduola AM. Potential contribution of prescription practices to the emergence and spread of chloroquine resistance in south west Nigeria:Caution in the use of artemisinim combination therapy. Malaria. Journal. 2009; 9-8.

[17] Breman JG, Alilio MS, Mills A. Conquering the intolerable burden of malaria: What's new, what's needed: A summary. American Journal of Tropical Medicine and Hygiene. 2004; 71(2): 1-15.

[18] World health organization. World malaria report [Internet]. Geneva. 2010. Available from https://who.int/malaria/world_malaria_report_2010/en/ 
[19] World health organization. Guidelines for Treatment of Malaria.Geneva. 2010.

[20] Federal Ministry of Health. National Malaria Control Programme [Internet]. Abuja, Nigeria, Strategic Plan.

[21] Okoro RN, Jamiu MO. The Cross-Sectional Evaluation of the Use of Artemisinin-Based Combination Therapy for Treatment of Malaria Infection at a Tertiary Hospital in Nigeria. Journal of Tropical Medicine. 2018; 2018: p. 6

[22] Tarning J. Treatment of Malaria in Pregnancy.The new England journal of medicine. 2016; 374: $981-982$.

[23] Federal Ministry of Health. National Malaria Control Programme, Abuja, Nigeria. Strategic Plan. 2008.

[24] Isah A, Isah R, Laing J, Quick et al. The Development of Reference Values for the WHO Health Facility Core Prescribing Indicators West African Journal of Pharmacology and Drug Research. 2001; 18: 1.

[25] Okoro RN, BG Shekari. Physicians' drug prescribing patterns at the national health insurance scheme unit of a teaching hospital in the North Eastern Nigeria," Archives of Pharmacy Practice. 2013; 4(1): 3.

[26] Fadare JA, Adeoti F, Aina O, Solomon, J Ijalana. The influence of health insurance scheme on the drug prescribing pattern in a Nigerian tertiary healthcare facility," Nigerian Medical Journal. 2015; 56(5): 344.

[27] Dodoo ANO, C Fogg, A Asiimwe et al. Pattern of drug utilization for treatment of uncomplicated malaria in urban Ghana following national treatment policy change to artemisinin-combination therapy, Malaria Journal. 2009; 8(1): 2.

[28] Marwaha N. Ascorbic acid co-administration with artemisinin based combination therapies in falciparum malaria .Indian J Med Res. 2016; 143(5): 539-541.

[29] LI I, Yan-Dan SU, Ming LI, Cheng-Gang ZOU. Hemin-mediated Hemolysis in Erythrocytes: Effects of Ascorbic Acid and Glutathione Shu-De Acta Biochimica et Biophysica Sinica. 2006; 38(1): 63-69. 